\title{
Finite-size effects in the absorption spectra of a single-wall carbon nanotube
}

\author{
Jaime Silva, ${ }^{*, \dagger}$ Micael J. T. Oliveira, ${ }^{*, \ddagger}$ Senentxu Lanceros-Mendez, ${ }^{*, \mathbb{I}, \S, \|}$ \\ and Fernando Nogueira*, $\dagger$ \\ $\dagger$ CFisUC, Department of Physics, University of Coimbra, Rua Larga, 3004-516 Coimbra, \\ Portugal. \\ $\$$ Department of Physics, University of Liège, B-4000, Liège, Belgium \\ IICenter of Physics, University of Minho, Campus de Gualtar, 4710-057 Braga, Portugal \\ $\S$ BCMaterials, Parque Científico y Tecnológico de Bizkaia, 48160-Derio, Spain \\ ||IKERBASQUE, Basque Foundation for Science, Bilbao, Spain \\ E-mail: silva.jaime@gmail.com; mjt.oliveira@ulg.ac.be; lanceros@fisica.uminho.pt; \\ fnog@uc.pt \\ Phone: +351239410681; +351239410114
}

\section{Abstract}

The determination of the optical spectrum of single-wall carbon nanotubes (SWCNTs) is essential for the development of opto-electronic components and sensors with application in many fields. Real SWCNTs are finite, but almost all the studies performed so far use infinite SWCNTs. However, the spectra of finite and infinite systems are different. In this work the optical spectrum of finite $(3,3)$ and $(5,5)$ SWCNTs is calculated as a function of nanotube length. For the $(3,3)$ SWCNTs, the calculated absorption spectra for light polarised both parallel and perpendicularly to the nanotube axis are in good agreement with experimental results. However, our results indicate that the lowest energy peak present in the experimental results for light polarised parallel to the nanotube axis can be attributed to a surface-plasmon resonance that is a consequence of the finite nature of the SWCNTs and not to the presence of SWCNTs with other chiralities, as claimed by the previous theoretical works. The surface-plasmon resonance is also studied using the Aharonov-Bohm effect. Finally, this work demonstrates that the surface-plasmon resonance in finite SWCNT can be described using a $1 \mathrm{D}$ infinite well.

\section{Introduction}

Historically, carbon nanotubes (CNTs) had their first appearance fifty years ago as undesirable byproducts of the fuel coke industry 1 . The subsequent detailed characterisation presented in the seminal work of Iijima ${ }^{2}$ for multiwall carbon nanotubes (MWCNTs) and, in a later work, for single wall carbon nanotubes (SWCNTs) ${ }^{3}$, drew the scientific community's attention to these carbon allotropes ${ }^{4.5}$. CNTs can be considered as cylinders of covalently bonded carbon atoms ${ }^{4}$ that, depending on how the graphene honeycomb network is rolled up, can be either metallic or semiconducting. The application range for CNTs is vast and includes, among others, conductive and highstrength composites, energy storage and energy conversion devices ${ }^{6}$. For CNTs, and in particular for SWCNTs, the geometry of the SWCNT is related to how the graphene honeycomb is rolled up and so the SWCNT surface can be specified by a pair of integer numbers $(m, n)$ denoting the relative position $C_{h}=n a_{1}+m a_{2}$ of a pair of atoms in the graphene lattice, with $a_{1}$ and $a_{2}$ being unit vectors of the hexagonal honeycomb lattice ${ }^{5}$. An important question for both metrological and applicative matters is how the optical absorption spec- 
trum varies with the CNT chirality as was recently demonstrated experimentally by Vialla et $a l^{7}$.

Although, in general, CNTs available for experimental study are a mixture of CNTs with different sizes and chiralities, the study of straight SWCNTs with a well defined diameter was possible by using $\mathrm{AIPO}_{4}-5$ zeolite to align in their channels a SWCNT with a $4 \AA$ diameter. Li et al ${ }^{8}$ used this method to measure the polarised absorption spectra of SWCNTs arrayed in the channels of the $\mathrm{AIPO}_{4}-5$ zeolite and drew the attention to the study of SWCNTs with a well defined diameter but unknown or unspecified chirality. The potential SWCNT candidates for such a small diameter are the $(3,3),(4,2)$ and $(5,0)$ SWCNTs. Li et al $\underline{8}$ reported that, when the exciting pulse electric field is polarised parallel to the SWCNT axis, a sharp peak at $1.37 \mathrm{eV}$ and two broad bands at 2.1 and $3.1 \mathrm{eV}$ are observed. The same results were also observed by Tang et $a l^{9}$. It was also demonstrated that the SWCNTs are transparent, due to a depolarisation effect, for light polarised perpendicularly to the SWCNT direction. The peaks' positions were explained through $a b$ initio calculations using the plane-wave pseudo-potential formulation 10,11 within the framework of the Local Density Approximation (LDA) of Density-Functional Theory (DFT) ${ }^{12,13}$ and their origin is traced back to transitions between bonding and antibonding $\pi$ orbitals.

Liu et $a l^{14}$, using another local DFT method ${ }^{15}$, calculated the density of states (DOS), the band structure and the absorption spectra of $(3,3),(4,2)$ and $(5,0)$ SWCNTs oriented parallel to the light polarisation axis. They also reported that the $(5,0)$ and $(3,3)$ SWCNTs are metallic while the $(4,2)$ is a small band gap semiconductor. The electronic properties, namely the DOS and the band structure of $(3,3),(4,2)$ and $(5,0)$ SWCNTs, were also addressed by Cabria et al ${ }^{16}$.

Machón et al 17 performed LDA calculations $\frac{18,19}{}$ of the structural, electronic, and optical properties of a $4 \AA$ SWCNT. When compared to the experimental work of $\mathrm{Li}$ et al ${ }^{8}$, the calculated optical properties revealed a discrepancy in the spectra for light polarised perpendicularly to the SWCNT axis: the SWCNTs were not transparent in the visible region. Li et al ${ }^{8}$ justified the disagreement by noting that the influence of the ze- olite on the optical properties had not been taken into account. This disagreement of the theoretical and experimental optical absorption spectra for light polarised perpendicularly to the SWCNT axis was explained by Marinopoulos et $a l^{20}$. These authors observed that theoretical studies of the optical spectra of SWCNT had been neglecting crystal local-field effects. By performing TimeDependent DFT (TDDFT) 21,22 calculations they were able to reproduce the experimental spectra of Li et al . However, the CNTs considered were infinite (with different chiralities), being therefore different from the ones used in the experiments. In this work we present a study of the absorption spectra of finite SWCNTs of different lengths and further clarify the experimental results of Li et $a l^{8}$.

\section{Results and discussion}

As previously mentioned, TDDFT has already been successfully applied to the calculation of the electronic excitations of SWCNTs. In this work we used the real-space code OCTOPUS ${ }^{23}$ to obtain the TDDFT optical spectra of finite $(3,3)$ and $(5,5)$ SWCNTs in the adiabatic TDLDA framework. The finite SWCNTs were generated 1 with different $k$ translations of the unit cell along the tube orientation, resulting in different SWCNT lengths as can be seen in Table 1 .

Table 1: Table with with different used $k$ translations and associated SWCNT length

\begin{tabular}{c|c|c}
$k$ & $\begin{array}{c}\text { Length }(\AA) \\
\text { SWCNT@ }(3,3)\end{array}$ & $\begin{array}{c}\text { Length }(\AA) \\
\text { SWCNT@ }(5,5)\end{array}$ \\
\hline 5 & 13.22 & 13.28 \\
10 & 26.19 & 26.23 \\
15 & 39.00 & 39.19 \\
20 & 51.58 & 50.92 \\
25 & 63.90 & 63.00 \\
30 & 76.28 & 78.00
\end{tabular}

We used the default Troullier-Martins pseudopotentials ${ }^{24}$ distributed with OCTOPUS and a uni-

\footnotetext{
${ }^{1}$ TubeGen 3.4 (web-interface, http://turin.nss.udel.edu/research/tubegenonline.html), J. T. Frey and D. J. Doren, University of Delaware, Newark DE, 2011
} 
form grid spacing of $0.20 \AA$. The simulation box was constructed joining spheres of radius $3.5 \AA$ around every atom. The time step used in the realtime propagation of the time-dependent KohnSham equations was $0.000658 \mathrm{fs}$, ensuring the stability of the time propagation. The terminations of the finite SWCNT were passivated with hydrogens and the entire structure was relaxed using a molecular dynamics code with the Merck molecular force field (MMFF94) $25,26$.

For the infinite $(3,3)$ SWCNT the code ABINIT $^{27}$ was used, also with the LDA approximation for exchange and correlation and Troullier-Martins pseudo-potentials. The infinite SWCNT was placed inside a parallelepipedic box of $(25,25,2.464282) \AA$. The plane-wave kinetic energy cutoff used was $35 \mathrm{Ha}$, with a kpoint Monkhorst-Pack mesh containing $(1,1,512)$ points.

The absorption spectra for incident light polarised perpendicularly to the $(3,3)$ and $(5,5)$ SWCNT axis for finite SWCNTs of different lengths were calculated and the results are shown in Fig. 1. The spectra are essentially the same, showing that the SWCNTs are transparent until around $4 \mathrm{eV}$, in good agreement with the experimental work of Li et $a l^{8}$ and Tang et $a l^{9}$ for the $(3,3)$ SWCNTs. The position of the first large peak is also in agreement with the results of the TDDFT calculations of Marinopoulos et $a^{20}$ for the $(3,3)$ SWCNTs. The increase in the height of the peaks with increasing SWCNT length is simply a reflection of the increase in the number of electrons that are available to be excited due to the increase in the number of carbon atoms.

In Fig. 2 the absorption spectra for light polarised parallel to the above mentioned $(3,3)$ and $(5,5)$ SWCNT axes are shown. For the $(3,3)$ SWCNTs there is a peak between 1.11 and $0.59 \mathrm{eV}$, depending on nanotube length, a second peak between 3.99 and $3.5 \mathrm{eV}$. As in the case of perpendicularly polarised light, the height of the peaks increases with increasing SWCNT length. Furthermore, the first two peaks can also be observed for the $(5,5)$ SWCNT at similar energies (and a third one at higher energies).

It is apparent from Fig. 2 that increasing the length of the SWCNT redshifts the lowest peaks, as expected for finite systems. This can be traced
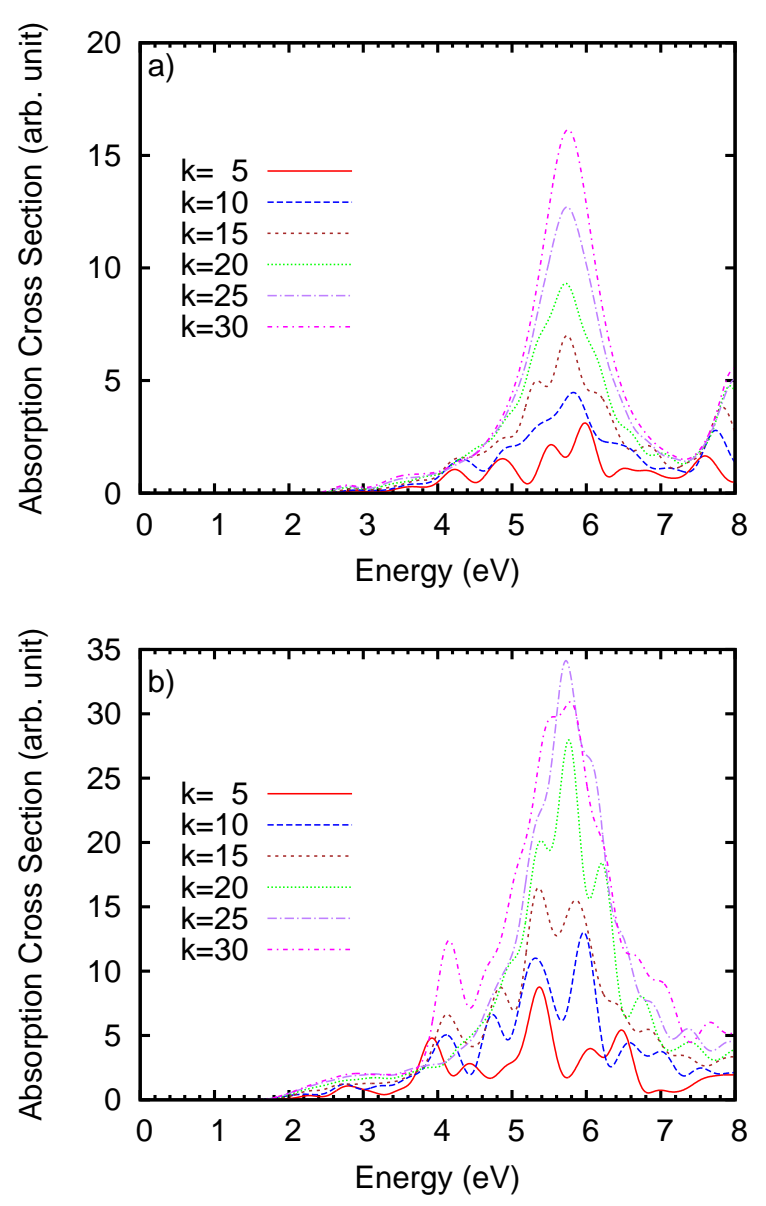

Figure 1: Absorption spectra of finite $(3,3)$ (a)) and $(5,5)$ (b)) SWCNTs of different lengths for incident light polarised perpendicularly to the SWCNT axis.

back to the fact that an approximated $f_{\mathrm{xc}}$ kernel (as the TDLDA used in this work) neglects dynamical correlations effects $\stackrel{28}{ }$. Nevertheless, it can be also attributed to a surface-plasmon resonance as it can be observed for simple atom chains ${ }^{29}$. Later in the text it is demonstrated that the first peak can indeed be attributed to a surface-plasmon resonance.

A notable difference between this work and previous ones is that the first peak observed in Fig. 2 is not present in the infinite $(3,3)$ SWCNT calculations as, e.g., those of Marinopoulos et $a l^{20}$ and Tang et $a l^{9}$, although it does appear in the experimental results of $\mathrm{Li}$ et $a l^{\frac{8}{}}$ and Tang et $a l^{9}$. In previous theoretical/computational works the presence of this peak was explained assuming the presence of SWCNTs with different chiralities, namely the $(3,3),(4,2)$, and $(5,0)$ SWCNTs. Our results suggest that the absence of the peak in previous calculations is related to the infinite nature of the 

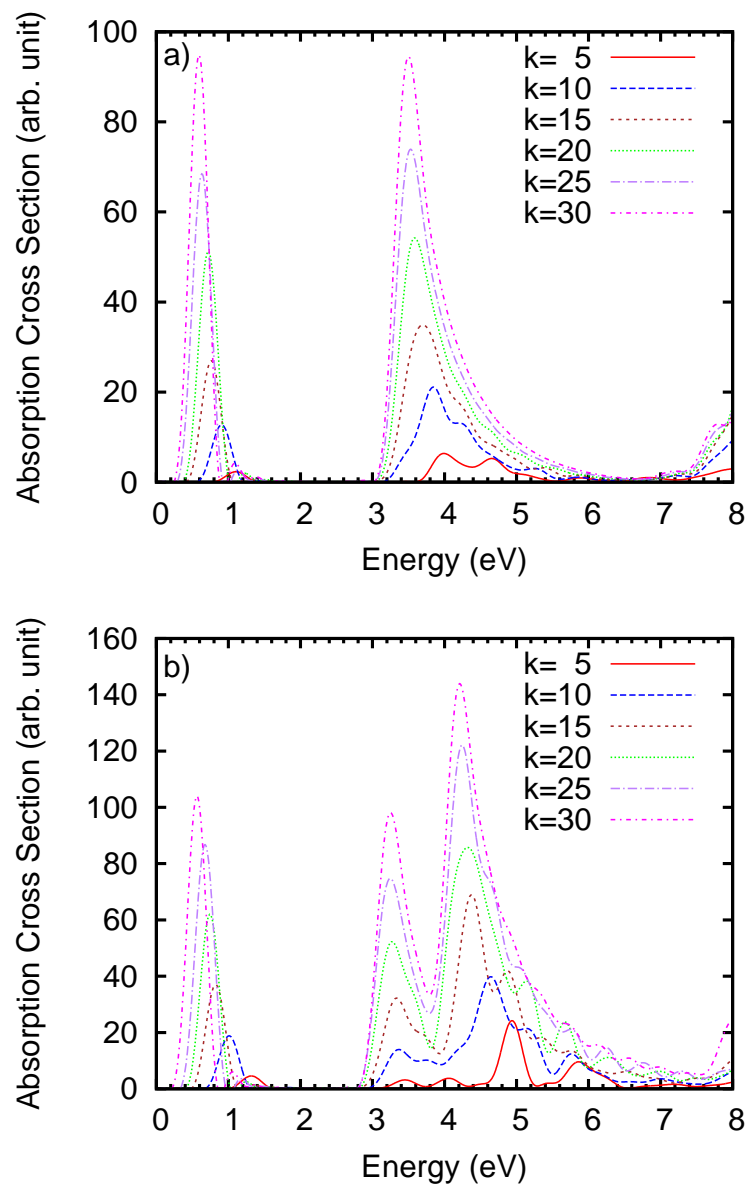

Figure 2: Absorption spectra of finite (3,3) (a)) and $(5,5)$ (b)) SWCNTs of different lengths for incident light polarised parallel to the SWCNT axis.

SWCNTs used. Our finite SWCNTs calculations do show that the peak should be present in samples consisting exclusively of $(n, n)$ SWCNTs like the $(3,3)$ and $(5,5)$ nanotubes. This claim would be impossible to check without a way of preparing SWCNT samples with a single chirality. Fortunately, the recent work of Blancon et al ${ }^{30}$ opened up this possibility, allowing our claim to be verified.

One straight explanation for the origin of the first peak observed in Fig. 2, is that it can be due to states localised at the ends of the SWCNT. To demonstrate the latter possibility, the optical spectra presented in Fig. 3, for incident light polarised parallel to the SWCNT, was calculated for the $(3,3)$ SWCNT with $k=5$ with closed ends (the open-ends SWCNT was terminated with carbon atoms), using the same methodology as described previously.

As can be seen in Fig. 3, when comparing open

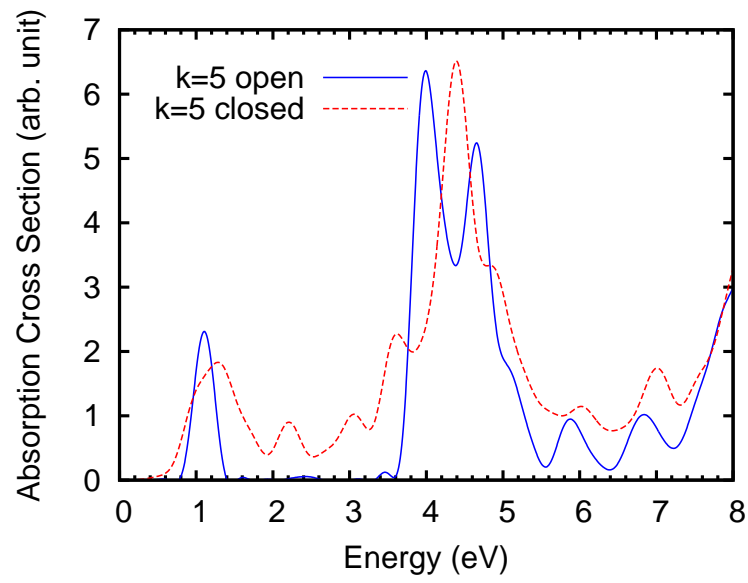

Figure 3: Absorption spectra of finite $(3,3)$ SWCNTs of length $k=5$ for incident light polarised parallel to the SWCNT axis with open and closed ends, terminated with carbon atoms.

and closed ends SWCNTs, the low energy peak is in the same position, but is broader for the closed SWCNT. This is due to the additional set of carbon atoms at both ends. Therefore, the origin of this peak can not be transitions involving localised states near the ends of the SWCNT. But the logical explanation for the absence of these peaks from the infinite SWCNT calculations is that the KohnSham states involved in this low-energy excitation are present in the finite SWCNTs but absent in the infinite one. Due to the size of the systems, the number of states present in them is too large to allow for an easy understanding of the origin of the peak. We therefore decided to look for the relevant states putting them in resonance with an applied laser. We studied the effect of an incident laser, polarised parallel to the SWCNT axis, with a frequency equal to the first peak of the $(3,3)$ SWCNT with $k=20(0.79 \mathrm{eV})$, a pulse width of $32.91 \mathrm{fs}$ and an enveloping cosine function. This study was performed with the OCTOPUS code for all the finite SWCNTs considered above. The resonant states were found by plotting the probability density for each state, after the application of the laser, and observing which states were disturbed by it. Combining this information with the density of states (DOS) of each nanotube readily highlights the difference between the finite and infinite nanotubes, as can be seen in Fig. 4. In this figure, the DOS for the different finite SWCNT lengths was normalised to the number of electrons of each nan- 

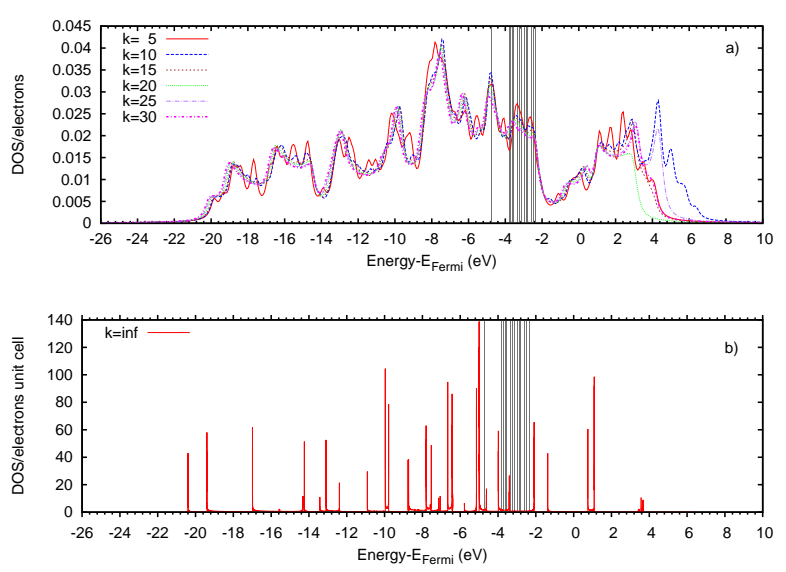

Figure 4: a) Normalised DOS for finite SWCNTs and b) for an infinite SWCNT. The grey vertical lines indicate the states that contribute to the first peak of the absorption spectra of the finite SWCNTs. The Fermi level was set at $0 \mathrm{eV}$ in both cases.

otube. The grey vertical lines indicate the resonant states found with the above described procedure (with a linewidth of $0.04 \mathrm{eV}$ ). The analysis of this figure clearly shows that there are states which are present in the finite nanotubes but disappear when the nanotube becomes infinite. These states are the ones contributing to the lowest energy peak, present in our calculations and on the experimental results of Li et $a l^{8}$, but not observed in the infinite nanotube calculations of Refs. ${ }^{20}$ and ${ }^{9}$. Note that the large differences above $3 \mathrm{eV}$ in Fig. $4 \mathrm{a}$ ) are simply due to the different number of unoccupied states considered in the calculations. Moreover, this nanotube is metallic and exhibits states around the Fermi level. These states are difficult to observe in the plot due to the large number of van Hove singularities.

From the results presented previously it is possible to state that this first peak is a surface-plasmon resonance and that its origin is the finite nature of the SWCNT. The confinement of the wave function on the finite 1D SWCNT leads to a standing wave and that shows up in the spectra as a surfaceplasmon resonance peak. This can be replicated with a set of independent particles that are confined in a $1 \mathrm{D}$ infinite well that has a length equal to the SWCNT. In fact, Fig. 5 presents the absorption spectra for a set of independent particles, $N=$ $20,70,100$, in a $1 \mathrm{D}$ infinite well with length equal to the SWCNTs with, respectively, $k=20,25,30$.
The TDDFT calculation was done using the exact same parameters, although the electron-electron interaction terms in the Hamiltonian (Hartree, exchange and correlation potentials) were set to zero.

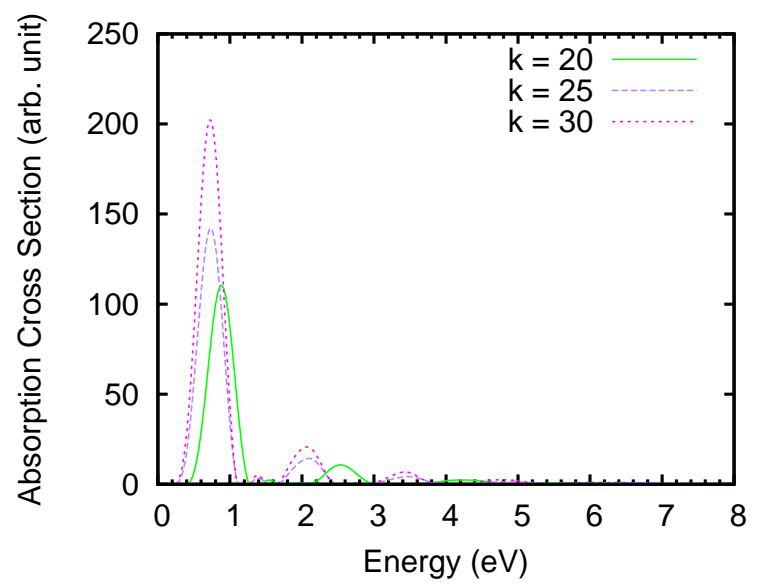

Figure 5: Absorption spectra for a set of independent particles in a $1 \mathrm{D}$ infinite well with length equal to the $(3,3)$ SWCNT

By comparing Fig. 5 with Fig. 22 it is possible to observe that the positions of the first peaks are similar. Furthermore, the origin of the small peaks that appear when the length of the SWCNT increases can be explained by the dependence of the single particle transition energies on the length of the 1D infinite well or SWCNT. When the SWCNT length increases the energy of the single particle transitions decreases, appearing as a peak at lower energies. Moreover, it was predicted by Nakanishi et $a l^{31}$ that, at the far infrared regime, the absorption spectra should exhibit a surface plasmon resonance peak. The authors of this work use a self consistent method that depends on an initial, frequency dependent, incident electric field and on the non-local conductivity of the nanotube. The predicted far infrared surface plasmon resonance peak was recently observed experimentally 32 for SWCNTs that have a length of the order of $1 \mu \mathrm{m}$. We stress that in our work the origin of the surface plasmon is distinctly different from the original proposal by Nakanishi et $a l^{31}$, i. e., in our work the calculations are $a b$-initio and do not depend on a particular electric field wavelength.

This lower energy peak has been observed in previous experimental work. The experimental spectra of Li et $a l^{\frac{8}{}}$ and Tang et $a l^{9}$ for light polarised parallel to the nanotube axis show a peak 


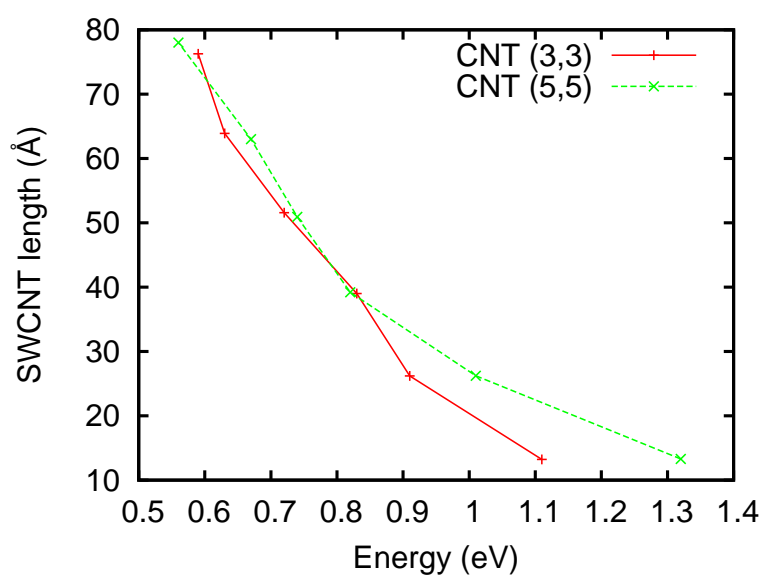

Figure 6: Lenght of the SWCNT versus the energy of the first peak for two types of armchair SWCNT.

at $1.37 \mathrm{eV}$ and two broad bands centred at 2.1 and $3.1 \mathrm{eV}$. Comparing these results to those presented in Fig. 2, it is apparent that the first peak of Fig. 2 is redshifted with respect to the first experimental peak and the second peak is blue-shifted relative to the third experimental peak. The broad experimental peak centred at $2.1 \mathrm{eV}$, however, is not present in our results. Previous attempts to theoretically reproduce the experimental results, like the work of Marinopoulos et al ${ }^{20}$, Machon et al ${ }^{17}$ and Spataru et al ${ }^{33}$, required the conjugation of the absorption spectra of three SWCNT with different chiralities. Moreover, as demonstrated by Spataru et $a l^{33}$ for the $(3,3)$ SWCNT, there is only one bound exciton that positions the third peak of the $(3,3)$ absorption spectra at $3.17 \mathrm{eV}$.

Another important question is how the energy of the first peak evolves with the increase in length of the SWCNT. Fig. 6 is a plot of the energy of the first peak versus the length of the tube for the two types of SWCNT, $(3,3)$ and $(5,5)$.

It is possible to observe that for the low aspect ratio $(3,3)$ and $(5,5)$ SWCNTs the energy of the first peaks of the $(3,3)$ tubes differs from that of the $(5,5)$ tubes. The latter is due to curvature effects (curvature is so strong that some rehybridization among the sigma and pi states appears) that alters the electronic structure of the nanotube and is more pronounced small diameter $\mathrm{SWCNT}^{5}$ like the $(3,3)$ SWCNT. Nevertheless, with the increase of SWCNT length, the energies of the first peaks are similar for the two types of armchair SWCNT, which is consistent with the 1D infinite well approximation. Moreover, the results presented in Fig. 6 do not follow a linear relation and, as expected for a surface plasmon resonance peak, the position of the peak depends on the length of the SWCNT. The presence of surface plasmon peak at low energies enables the use of SWCNT as plasmonics-active systems ${ }^{34}$. The application of the surface plasmon resonance is vast and can be use in bioanalytic and biophysical applications 35 . In fact for SWCNT there is increase interest in studying the influence of the surface plasmon resonance with SWCNT physical properties as is demonstrated, for higher frequency plasmon, in this recent work 36 . Finally, it is important to point that TDDFT was employed to study the surface plasmon resonance of other systems 37

It is well known that, when modelling a finite portion of an infinite metallic nanotube, the HOMO-LUMO gap usually opens. Low-energy peaks could be attributed to this gap opening. However, in this study the gap is very small and this explanation for the low-energy peaks can be safely discarded.

Nevertheless, one can test the effect of the HOMO-LUMO gap opening in the absorption spectra of a finite $(5,5)$ CNT using the AharonovBohm effect ${ }^{38}$. For that we add to the normal DFT/TDFT calculations a static magnetic field parallel to the CNT main axes with a value, for the $(5,5)$ CNT with $k=15$, between 1043.4 and $1.3564 \times 10^{4}$ Tesla. In a celebrated work, $\underline{38}$ Aharonov and Bohm describe a possible experiment to study the quantum effects of the application of a potential to charged particles. In their work 38 it is described an effect that arises from the quantum nature of the matter, i. e., a charged particle feels the effect of a potential that was acting on it even if there is no field in the current region. To explain the latter effect, Aharonov and Bohm proposed a hypothetical experiment where it is demonstrated that, by using a magnetic flux in a selected region of space, two electron beams acquire a phase difference that is proportional to the magnetic flux quantum, $\phi_{0}=\frac{h}{e}$ with $h$ as the Planck constant and $e$ the electronic charge, even if the magnetic flux has a zero value in the region where the measurement is performed. The Aharonov-Bohm effect ${ }^{38}$ was demonstrated experimentally by Tonomura et al. ${ }^{39}$ in 1986 start- 
ing the application and study of this effect to meso and nano systems. For infinite CNT, the application of the Aharonov-Bohm effect results in oscillations of the band gap first described by Ajiki and Ando ${ }^{40,41}$. Furthermore, in the case of the metallic CNT, there is a gap opening that is dependent on the applied magnetic flux and has $\phi_{0}$ periodicity 40,41 . More recently it has been shown that curvature effects break the periodicity of the gap oscillation $^{42}$. In Fig. 7 it is presented the absorption cross section versus applied magnetic flux for the $(5,5) \mathrm{CNT}$. It is possible to observe the existence of two type of peaks. The first one, the plasmonic peak, oscillating with the increase of the applied magnetic flux, and the usual metallic peak splitting with the application of magnetic flux.

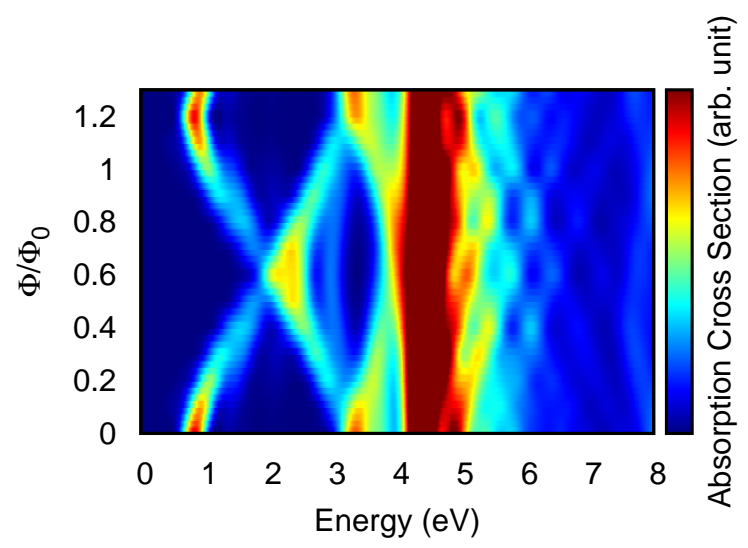

Figure 7: Absorption cross section versus applied magnetic flux for the $(5,5) \mathrm{CNT}$.

As previously explained, with the increase of the magnetic flux the band gap oscillates and, for finite nanotubes, the energy difference between the highest occupied molecular orbital (HOMO) and the lowest unoccupied molecular orbital (LUMO) also oscillates with a periodicity higher than $\phi_{0}$, as can be seen in Fig. 8 This oscillation of the HOMO - LUMO gap is at the origin of the plasmonic peak position with the increase of the magnetic flux. This is due to the fact that the peak is only related with independent particles transitions in a confined environment and so it depends on the energy difference between the occupied an unoccupied orbitals. In the same figure it is possible to observe that the metallic peak splits with the increase of the magnetic flux. The splitting can be explained for infinite CNT with the lifting of the van Hove singularities ${ }^{5,43}$. For the finite CNT the metallic peaks spliting is related to the lifting of degenerated molecular orbitals, as can be seen observed in figure Fig. 8 .

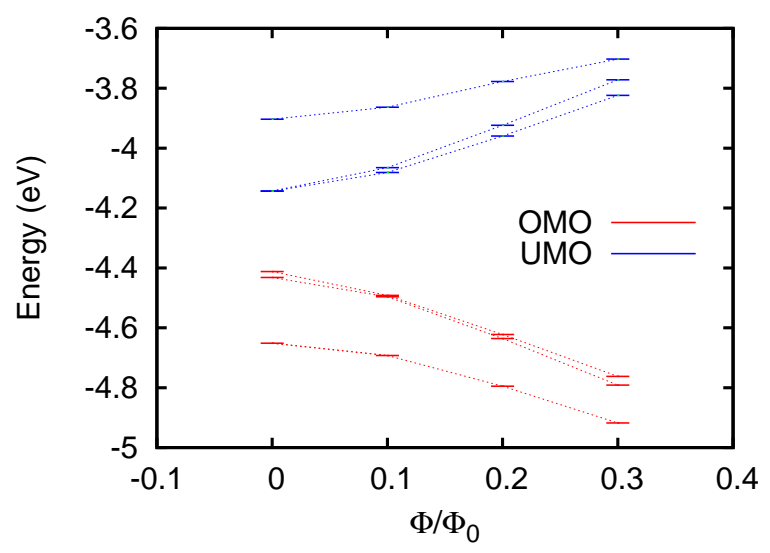

Figure 8: Occupied molecular orbitals (OMO) and unoccupied molecular orbitals (UMO) energies versus magnetic flux for the $(5,5) \mathrm{CNT}$, showing the HOMO and LUMO followed by the two immediately molecular orbitals.

It can also be seen in Fig. 7 that the surface plasmon peak does not split, hence the origin of the plasmon peak is not related to transitions from/to degenerated molecular orbitals or, in the case of infinite CNT, from/to the van Hove singularities, as demonstrated previously. Another point, is the periodicity of the HOMO-LUMO gap presented in Fig. 9, as it can be seen that the period is greater than $\phi_{0}$.

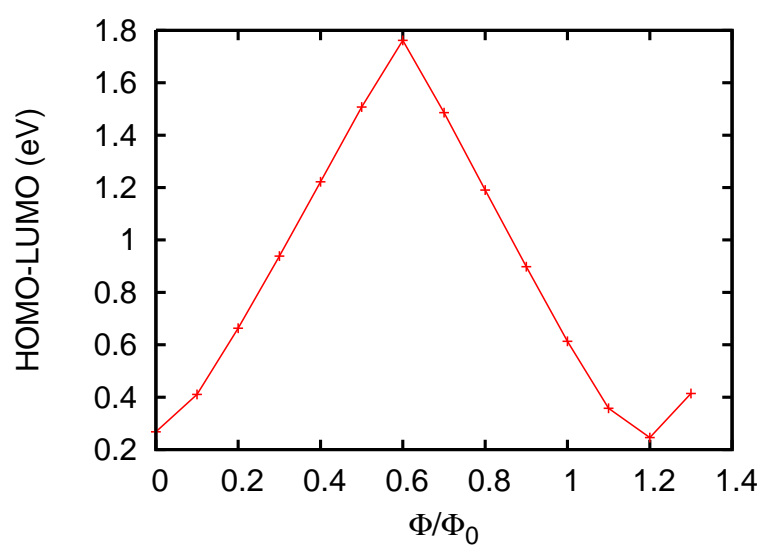

Figure 9: HOMO-LUMO difference versus magnetic flux for the $(5,5) \mathrm{CNT}$.

This anomalous Aharonov-Bohm effect, as ex- 
plained by Sangalli et. al. ${ }^{42}$, is related to curvature effects or to impurities and defects that creates deviations from a perfect circle of radius $R_{C N T}$. In fact, by employing an optimization procedure to relax the finite CNT structures produces oscillations on the value of $R_{C N T}$, mainly at the ends of the CNT.

\section{Conclusions}

In this work the optical spectra of finite $(3,3)$ and $(5,5)$ SWCNTs with different lengths were calculated. It was found that these nanotubes are transparent to visible and near-UV light polarised perpendicularly to their axis, in agreement with previous experimental and theoretical results. It was also shown that the absorption spectra for light polarised parallel to the nanotubes' axes exhibit a low energy peak that is redshifted with the increase of the SWCNT length. This peak is present in previous experimental studies, and our results suggest that its absence from previous calculations is related to the infinite nature of the SWCNTs used in those and not to the presence of nanotubes with other chiralities, as previously thought. The method recently proposed by Blancon et al ${ }^{30}$ will allow for the validation of this claim. This low energy peak is studied using the Aharonov-Bohm effect in CNT confirming that it is a plasmonic peak energy and is due to the finite nature of CNT and also that it can be described using 1D infinite well. Finally the relation between the plasmonic peak position and the SWCNT length is also a signature 1D infinite well. Nevertheless, for the longer SWCNT (longer than the SWCNT study in this work) other effects must be considered like the van der Waals interaction.

\section{Acknowledgments}

We thank the Laboratory for Advanced Computing (LCA) of the University of Coimbra for the computer time allocated to this project. 


\section{References}

(1) Monthioux, M. Filling Single-Wall Carbon Nanotubes. Carbon 2002, 40, 1809-1823.

(2) Iijima, S. Helical Microtubles of Graphitic Carbon. Nature 1991, 354, 56-58.

(3) Iijima, S.; Ichihashi, T. Single-shell Carbon Nanotubes of 1-nm Diameter. Nature 1993, 363, 603-605.

(4) Moniruzzaman, M.; Winey, K. I. Polymer Nanocomposites Containing Carbon Nanotubes. Macromolecules 2006, 39, 51945205.

(5) Charlier, J.-C.; Blase, X.; Roche, S. Electronic and Transport Properties of Nanotubes. Rev. Mod. Phys. 2007, 79, 677-732.

(6) Baughman, R. H.; Zakhidov, A. A.; de Heer, W. A. Carbon Nanotubes-the Route Toward Applications. Science 2002, 297, 787-792.

(7) Vialla, F.; Roquelet, C.; Langlois, B.; Delport, G.; Santos, S. M.; Deleporte, E.; Roussignol, P.; Delalande, C.; Voisin, C.; Lauret, J.-S. Chirality Dependence of the Absorption Cross Section of Carbon Nanotubes. Phys. Rev. Lett. 2013, 111, 137402.

(8) Li, Z.; Tang, Z.; Liu, H.; Wang, N.; Chan, C.; Saito, R.; Okada, S.; Li, G.; Chen, J.; Nagasawa, N. et al. Polarized Absorption Spectra of Single-Walled $4 \AA$ Carbon Nanotubes Aligned in Channels of an AlPO4-5 Single Crystal. Phys. Rev. Lett. 2001, 87, 127401.

(9) Tang, Z. K.; Wang, N.; Zhang, X. X.; Wang, J. N.; Chan, C. T. Novel Properties of $0.4 \mathrm{~nm}$ Single-Walled Carbon Nanotubes Templated in the Channels of AlPO4-5. New J. Phys. 2003, 5, 146.1-146.29.

(10) Kresse, G.; Hafner, J. Ab Initio Molecular Dynamics for Liquid Metals. Phys. Rev. B 1993, 47, 558-561.

(11) Kresse, G.; Furthmüller, J. Efficiency of AbInitio Total Energy calculations for Metals and Semiconductors Using a Plane-Wave Basis Set. Comput. Mater. Sci. 1996, 6, 15-50.

(12) Hohenberg, P.; Kohn, W. Inhomogeneous Electron Gas. Phys. Rev. 1964, 136, B864B871.

(13) Kohn, W.; Sham, L. J. Self-Consistent Equations Including Exchange and Correlation Effects. Phys. Rev. 1965, 140, A1133A1138.

(14) Liu, H.; Chan, C. Properties of $4 \AA$ Carbon Nanotubes From First-Principles Calculations. Phys. Rev. B 2002, 66, 115416.

(15) Mintmire, J. W.; White, C. T. $X \alpha$ Approach for the Determination of Electronic and Geometric Structure of Polyacetylene and Other Chain Polymers. Phys. Rev. Lett. 1983, 50, 101-105.

(16) Cabria, I.; Mintmire, J.; White, C. Metallic and Semiconducting Narrow Carbon Nanotubes. Phys. Rev. B 2003, 67, 121406.

(17) Machón, M.; Reich, S.; Thomsen, C.; Sánchez-Portal, D.; Ordejón, P. Ab initio Calculations of the Optical Properties of 4$\AA$ Diameter Single-Walled Nanotubes. Phys. Rev. B 2002, 66, 155410.

(18) Sánchez-Portal, D.; Ordejón, P.; Artacho, E.; Soler, J. M. Density-Functional Method for Very Large Systems With LCAO Basis Sets. Int. J. Quantum Chem. 1997, 65, 453-461.

(19) Soler, J. M.; Artacho, E.; Gale, J. D.; García, A.; Junquera, J.; Ordejón, P.; SánchezPortal, D. The SIESTA Method for Ab Initio Order- N Materials Simulation. J. Phys.: Condens. Mat. 2002, 14, 2745-2779.

(20) Marinopoulos, A.; Reining, L.; Rubio, A.; Vast, N. Optical and Loss Spectra of Carbon Nanotubes: Depolarization Effects and Intertube Interactions. Phys. Rev. Lett. 2003, 91, 046402 .

(21) Runge, E.; Gross, E. K. U. DensityFunctional Theory for Time-Dependent Systems. Phys. Rev. Lett. 1984, 52, 997-1000. 
(22) Marques, M.; Maitra, N.; Nogueira, F.; Gross, E. K. U.; Rubio, A. Fundamentals of Time-Dependent Density Functional Theory; Lecture Notes in Physics; Springer Berlin Heidelberg, 2012.

(23) Andrade, X.; Alberdi-Rodriguez, J.; Strubbe, D. A.; Oliveira, M. J. T.; Nogueira, F.; Castro, A.; Muguerza, J.; Arruabarrena, A.; Louie, S. G.; Aspuru-Guzik, A. et al. Time-Dependent Density-Functional Theory in Massively Parallel Computer Architectures: the Octopus Project. J. Phys.: Condens. Mat. 2012, 24, 233202.

(24) Troullier, N.; Martins, J. L. Efficient Pseudopotentials for Plane-Wave Calculations. Phys. Rev. B 1991, 43, 1993-2006.

(25) Halgren, T. A. Merck Molecular Force Field. II. MMFF94 van der Waals and Electrostatic Parameters for Intermolecular Interactions. $J$. Comput. Chem. 1996, 17, 520-552.

(26) Halgren, T. A. Merck Molecular Force Field. I. Basis, Form, Scope, Parameterization, and Performance of MMFF94. J. Comput. Chem. 1996, 17, 490-519.

(27) Gonze, X.; Amadon, B.; Anglade, P.-M.; Beuken, J.-M.; Bottin, F.; Boulanger, P.; Bruneval, F.; Caliste, D.; Caracas, R.; Côté, M. et al. ABINIT: First-Principles Approach to Material and Nanosystem Properties. Comput. Phys. Commun. 2009, 180, 2582-2615.

(28) Onida, G.; Reining, L.; Rubio, A. Electronic Excitations : Density-Functional Versus Many-Body Green's-Function Approaches. Rev. Mod. Phys. 2002, 74, 601-659.

(29) Yan, J.; Gao, S. Plasmon Resonances in Linear Atomic Chains: Free-Electron Behavior and Anisotropic Screening of d Electrons. Phys. Rev. B 2008, 78, 235413.

(30) Blancon, J.-C.; Paillet, M.; Tran, H. N.; Than, X. T.; Guebrou, S. A.; Ayari, A.; Miguel, A. S.; Phan, N.-M.; Zahab, A.-A.;
Sauvajol, J.-L. et al. Direct Measurement of the Absolute Absorption Spectrum of Individual Semiconducting Single-Wall Carbon Nanotubes. Nat. Commun. 2013, 4, 2542.

(31) Nakanishi, T.; Ando, T. Optical Response of Finite-Length Carbon Nanotubes. J. Phys. Soc. Jpn. 2009, 78, 114708.

(32) Morimoto, T.; Joung, S.-K.; Saito, T.; Futaba, D. N.; Hata, K.; Okazaki, T. Lengthdependent plasmon resonance in singlewalled carbon nanotubes. ACS Nano 2014, 8 , 9897-9904.

(33) Spataru, C.; Ismail-Beigi, S.; Benedict, L.; Louie, S. Excitonic Effects and Optical Spectra of Single-Walled Carbon Nanotubes. Phys. Rev. Lett. 2004, 92, 077402.

(34) Vo-Dinh, T.; Dhawan, A.; Norton, S. J.; Khoury, C. G.; Wang, H.-N.; Misra, V.; Gerhold, M. D. Plasmonic Nanoparticles and Nanowires: Design, Fabrication and Application in Sensing. J. Phys. Chem. C 2010, 114, 7480-7488.

(35) Couture, M.; Zhao, S. S.; Masson, J.-F. Modern Surface Plasmon Resonance for Bioanalytics and Biophysics. Phys. Chem. Chem. Phys. 2013, 15, 11190-11216.

(36) Rance, G. a.; Marsh, D. H.; Nicholas, R. J.; Khlobystov, A. N. UVâĂŞVis Absorption Apectroscopy of Carbon Nanotubes: Relationship Between the $\pi$-Electron Plasmon and Nanotube Diameter. Chem. Phys. Lett. 2010, 493, 19-23.

(37) López-Lozano, X.; Barron, H.; Mottet, C.; Weissker, H.-C. Aspect-Ratio- and Size-Dependent Emergence of the SurfacePlasmon Resonance in Gold Nanorods-an Ab Initio TDDFT Study. Phys. Chem. Chem. Phys. 2014, 16, 1820-1823.

(38) Aharonov, Y.; Bohm, D. Further Considerations on Electromagnetic Potentials in the Quantum Theory. Phys. Rev. 1961, 123, 1511-1524. 
(39) Tonomura, A.; Osakabe, N.; Matsuda, T.; Kawasaki, T.; Endo, J.; Yano, S.; Yamada, H. Evidence for Aharonov-Bohm Effect With Magnetic Field Completely Shielded From Electron Wave. Phys. Rev. Lett. 1986, 56, 792-795.

(40) Ajiki, H.; Ando, T. Electronic States of Carbon Nanotubes. J. Phys. Soc. Jpn. 1993, 62, 1255-1266.

(41) Ajiki, H.; Ando, T. Aharonov-Bohm Effect in Carbon Nanotubes. Physica B 1994, 201, 349-352.

(42) Sangalli, D.; Marini, A. Anomalous Aharonov - Bohm Gap Oscillations in Carbon Nanotubes. Nano Lett. 2011, 11, 4052-4057.

(43) Roche, S.; Dresselhaus, G.; Dresselhaus, M. S.; Saito, R. Aharonov-bohm Spectral Features and Coherence Lengths in Carbon Nanotubes. Phys. Rev. B: Condens. Matter Mater. Phys. 2000, 62, 16092-16099. 


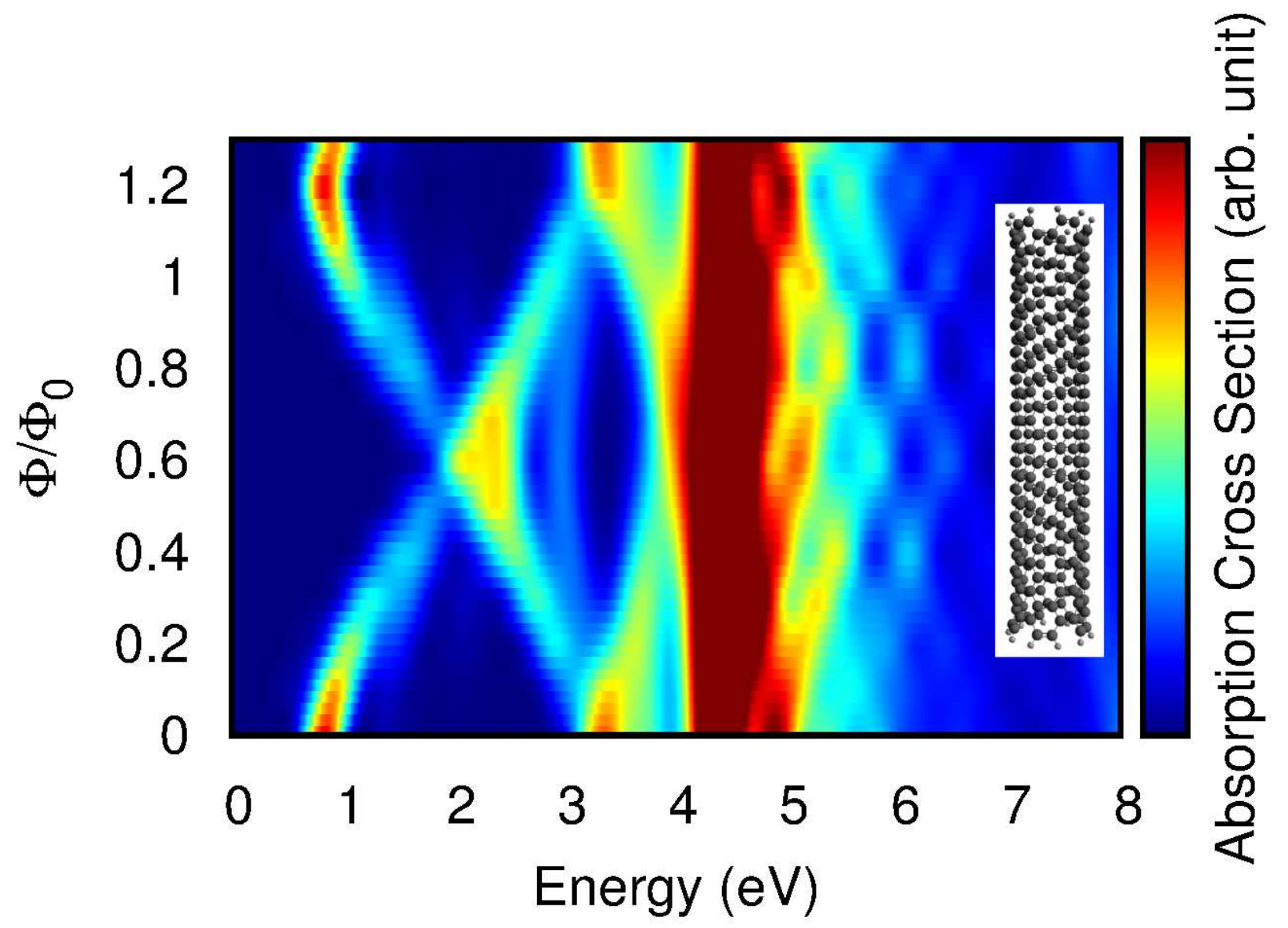

Figure 10: TOC. 